\title{
Uniform Attractor for A Non-autonomous Parabolic Equation with Nonlocal Diffusion and Delay
}

\author{
Miaomiao Wang, Weiwei Chang and Xiaojun $\mathrm{Li}^{*}$ \\ School of Science, Hohai University, Nanjing, Jiangsu 210098, China \\ Email: lixjun05@mailsvr.hhu.edu.cn
}

\begin{abstract}
This paper is devoted to study the long-time behavior of non-autonomous parabolic equation with nonlocal diffusion and hereditary effect, where time symbol is translation bound in $L_{l o c}^{2}\left(\mathbb{R} ; H^{-1}(\Omega)\right)$ and $L_{l o c}^{2}\left(\mathbb{R} ; L^{2}(\Omega)\right)$, respectively. By the energy estimates and asymptotic priori estimates of solutions, we obtain the existence and regularity of uniform attractor for the family of processes corresponding to original systems, respectively.
\end{abstract}

Keywords: Uniform attractor; delay; nonlocal diffusion; non-autonomous parabolic equation; uniform $\omega$-limit compact.

\section{Introduction}

Consider the following non-autonomous parabolic equation with nonlocal diffusion and delay effect

$$
\left\{\begin{array}{l}
\frac{\partial u}{\partial t}-a(l(u)) \triangle u=f(u)+g\left(u_{t}\right)+h(t) \quad \text { in } \quad \Omega \times(\tau, \infty), \\
u=0 \quad \text { on } \quad \partial \Omega \times(\tau, \infty), \\
u(x, \tau+s)=\varphi(x, s) \quad x \in \Omega, s \in[-k, 0], \tau \in \mathbb{R}
\end{array}\right.
$$

where $\Omega \subset \mathbb{R}^{N}$ is a bounded domain, $f \in C(\mathbb{R}), a \in C\left(\mathbb{R}, \mathbb{R}^{+}\right)$is locally Lipschitz, and there exist positive constants $m, M$ such that

$$
0<m \leq a(s) \leq M, \quad \forall s \in \mathbb{R} .
$$

In addition, $g$ is an operator satisfying some assumption which will be given below, the time-dependent force term $h$ belongs to $L_{l o c}^{2}\left(\mathbb{R} ; H^{-1}(\Omega)\right)$ and $L_{l o c}^{2}\left(\mathbb{R} ; L^{2}(\Omega)\right)$, respectively, and $\varphi$ belonging to $C([-k, 0]$; $\left.L^{2}(\Omega)\right)$ is the initial data, where $k>0$ is the length of the delay effect. For each $t \geq \tau$, we denote by $u_{t}$ the function defined on $[-k, 0]: u_{t}(s)=u(t+s), s \in[-k, 0]$.

In recent decades, the evolutionary equations with nonlocal diffusion and delay effect have been intensively studied because of their wide applications in Physics, Biology, Chemistry and many other fields. For example, in the semiconductor equations, the presence of the nonlocal term $a=a(l(u))$ can be used to describe the rate of diffusion of thermodynamics which depends on the nonlocal number (see,e.g., [1,2]). In Biological equation, as an example of a spatial nonlocal feature, the velocity of migration $\vec{v}=a \nabla u$ depends on the global population in a certain subdomain (see [3]). In addition, evolution equations with delay also have lots of applications. For example, time delay in the infectious disease model is represented as the incubation period of the disease in the crowd (see [4]). There are many mathematic research about the nonlocal and delay problem, see,e.g., [5-11].

In this paper, we study the dynamics of the non-autonomous evolution equation (1.1) with nonlocal diffusion and delay. When $a(s)=1$ or $g(s)=0$ for all $s$ belonging to $\mathbb{R}$, the pullback attractor of (1.1) was established in [10] and [7], respectively. Here, we establish the existence of uniform attractor for (1.1). When the uniform attractor exists, it includes the union of pullback attractor. The standard method in obtaining uniform attractor is of constructing skew-product flow. This method involves parameter spaces and requires that symbol is translation compact (see [12]). When the time symbol is only translation bounded, by the methods in $[13,14]$, we establish the existence of uniform attractor for $(1.1)$ in $C_{L^{2}}$ and $C_{L^{p}}$, respectively. 
Let $l \in\left(L^{2}(\Omega)\right)^{\prime}$. We make some assumptions on nonlinearity $f$. Suppose that there exist positive constant $\alpha_{1}, \alpha_{2}, c_{1}, \ell$, and $p>2$ such that

$$
\begin{aligned}
& -c_{1}-a_{1}|s|^{p} \leq f(s) s \leq c_{1}-a_{2}|s|^{p}, \quad \forall s \in \mathbb{R} \\
& (f(s)-f(r))(s-r) \leq \ell(s-r)^{2}, \quad \forall r, s \in \mathbb{R} .
\end{aligned}
$$

From (1.3), we deduce that there exists $c_{2}>0$ such that

$$
|f(s)| \leq c_{2}\left(|s|^{p-1}+1\right), \quad \forall s \in \mathbb{R} .
$$

Let

$$
F(s)=\int_{0}^{s} f(r) d r .
$$

From(1.3) we get that there exist positive constants $\widetilde{a_{1}}, \widetilde{a_{2}}$ and $\widetilde{c_{1}}$ such that

$$
-\widetilde{c_{1}}-\widetilde{a_{1}}|s|^{p} \leq F(s) \leq \widetilde{c_{1}}-\widetilde{a_{2}}|s|^{p}, \quad \forall s \in \mathbb{R} .
$$

For the operator $g$, we assume that $g: C_{L^{2}} \rightarrow L^{2}(\Omega)$ satisfies

(I) $g(0)=0$,

(II) there exists $L_{g}>0$ such that for all $t \in \mathbb{R}$ and $\xi, \eta \in C_{L^{2}}$,it holds

$$
|g(\xi)-g(\eta)| \leq L_{g}|\xi-\eta|_{C_{L^{2}}}
$$

where $L_{g}<m \lambda_{1}, \lambda_{1}$ is the first eigenvalue of $-\Delta$ in $H_{0}^{1}(\Omega)$.

As usual, we denote by $(\cdot, \cdot)$ and $|\cdot|$ the scalar product and norm in $L^{2}(\Omega)$, respectively; the norm in $L^{p}(\Omega)$ is written as $\|\cdot\|_{L^{p}(\Omega)}$; and we denote the inner product in $H_{0}^{1}(\Omega)$ by $((\cdot, \cdot))=(\nabla \cdot \nabla \cdot)$, with the associated norm $\|\cdot\|$. We also denote by $C_{L^{p}}$ the Banach space $C\left([-k, 0] ; L^{p}(\Omega)\right), p \geq 2$, equipped with the sup-norm, i.e., for any element $u \in C_{L^{p}}$, its norm is defined by $\|u\|_{C_{L^{p}}}=\max _{t \in[-k, 0]}\|u(t)\|_{L^{p}}$. The duality between $L^{p}(\Omega)$ and its dual $L^{q}(\Omega)$, where $q=p /(p-1)(1<p<\infty)$, is also denoted by $(\cdot, \cdot)$, and the duality between $H_{0}^{1}(\Omega)$ and its dual $H^{-1}(\Omega)$ is written as $\langle\cdot, \cdot\rangle$, and we denote by $\|\cdot\|_{*}$ the norm in $H^{-1}(\Omega)$.

The paper is organized as follows: In the next section, we establish the well-posedness of (1.1) and recall some fundamental results on existence of uniform attractor; in Section 3, we show the existence of uniform attractor for (1.1) in $C_{L^{2}}$; in Section 4, we show the existence of uniform attractor for (1.1) in $C_{L^{p}}$.

\section{Preliminaries}

The aim of this section is to give the existence of solution for (1.1) and recall some results on the theory of uniform attractors.

Definition 2.1. A weak solution to (1.1) with initial datum $\varphi \in C_{L^{2}}$ and $u(t)=\varphi(t-\tau)$ for all $t \in[\tau-k, \tau]$ is a function $u \in C\left([\tau-k, \infty] ; L^{2}(\Omega)\right)$ such that $u \in L^{2}\left(\tau, T ; H_{0}^{1}(\Omega)\right) \cap L^{p}\left(\tau, T ; L^{p}(\Omega)\right)$ for all $T>\tau$, and for all $v \in H_{0}^{1}(\Omega) \cap L^{p}(\Omega)$, it satisfies

$$
\frac{d}{d t}(u(t), v)+a(l(u(t)))((u(t), v))=(f(u(t)), v)+\left(g\left(u_{t}\right), v\right)+\langle h(t), v\rangle, \quad \forall v \in H_{0}^{1} \cap L^{p}(\Omega) .
$$

Remark 2.1. Observe that if $u$ is a weak solution to (1.1), then it satisfies the energy equality

$$
\begin{aligned}
|u(t)|^{2} & +2 \int_{s}^{t} a(l(u(r)))\|u(r)\|^{2} d r=|u(s)|^{2}+2 \int_{s}^{t}\left[(f(u(r)), u(r))+\left(g\left(u_{r}\right), u(r)\right)\right. \\
& +\langle h(r), u(r)\rangle] d r
\end{aligned}
$$

for all $\tau \leq s \leq t$. 
Next result gives the well-posedness of the system (1.1), the idea of the proof is close to that in $[7,10]$.

Theorem 2.1. Assume that the function a is locally lipschitz and satisfies (1.2), $f \in C(\mathbb{R})$ fulfills (1.3)-(1.4), $h \in L_{l o c}^{2}\left(\mathbb{R}, H^{-1}(\Omega)\right), l \in\left(L^{2}(\Omega)\right)^{\prime}, g$ satisfies (I)-(III), and $\varphi \in C_{L^{2}}$ is given. Then there exists a unique weak solution $u(\cdot)=u(\cdot ; \tau, \varphi)$ to (1.1).

Consider the following abstract non-autonomous evolution equation

$$
\partial_{t} u=A_{\sigma(t)}(u), \quad t \in \mathbb{R}
$$

where $A_{\sigma(s)}: E_{1} \rightarrow E_{0}, s \in \mathbb{R}$, is the evolutionary operator, $E_{0}$ and $E_{1}$ are Banach spaces, the $\sigma(s), s \in \mathbb{R}$, is a time-dependent function parameter which is called time symbol. The function value of $\sigma(s)$ belongs to some metric space (or Banach space) $\Xi$, i.e., for $\forall s \in \mathbb{R}, \sigma(s) \in \Xi$.

We impose the initial value to $(2.3)$ :

$$
\left.u\right|_{t=\tau}=u_{\tau}, u_{\tau} \in E,
$$

here $\mathrm{E}$ is a Banach space and satisfies $E_{1} \subseteq E \subseteq E_{0}$. Assume that for every $\sigma(s) \in \Sigma$, where $\Sigma \subset \Xi$ is a parameter set, and for $u_{\tau} \in E$, there exists a unique solution for (2.3)-(2.4). As a result, the solution of (2.3)-(2.4) can be described as a two-parameter family of operators:

$$
u(t)=U_{\sigma}(t, \tau) u_{\tau}, u_{\tau} \in E, \forall t \geq \tau, \sigma=\sigma(t) \in \Sigma, t, \tau \in \mathbb{R} .
$$

Definition 2.2. The two-parameter family of mappings $\left\{U_{\sigma}(t, \tau), t \geq \tau, \tau \in \mathbb{R}\right\}, \sigma \in \Sigma$, acting in the Banach space $E$ is said to be a family of processes with time symbol $\sigma \in \Sigma$ if for each $\sigma \in \Sigma$,

$$
U_{\sigma}(t, \tau): E \rightarrow E, \quad t \geq \tau, \tau \in \mathbb{R}
$$

and satisfies the following multiplicative properties:

$$
\begin{gathered}
U_{\sigma}(t, s) U_{\sigma}(s, \tau)=U_{\sigma}(t, \tau), \quad \forall t \geq s \geq \tau, \tau \in \mathbb{R}, \\
U_{\sigma}(\tau, \tau)=I d \text { is the identity operator, } \tau \in \mathbb{R} .
\end{gathered}
$$

Note that the following translation identity is valid for the family of process $U_{\sigma}(t, \tau), \sigma \in \Sigma$, generated by a problem, which is uniquely solvable, and for the translation semigroup $\{T(h): h \geq 0\}$ :

$$
U_{\sigma}(t+h, \tau+h)=U_{T(h) \sigma}(t, \tau), \forall \sigma \in \Sigma, t \geq \tau, \tau \in \mathbb{R}, h \geq 0 .
$$

$\left\{U_{\sigma}(t, \tau)\right\}$ is said to be weakly continuous in $(E \times \Sigma, E)$, if for each $t \geq \tau, \tau \in \mathbb{R},(u, \sigma) \rightarrow U_{\sigma}(t, \tau)$ is a weakly continuous map from $E \times \Sigma$ to $E$. Denote by $\mathcal{B}(E)$ the set of all bounded subsets of $E$.

Definition 2.3. A set $B_{0} \subset E$ is said to be a uniformly (w.r.t. $\sigma \in \Sigma$ ) absorbing set for $\left\{U_{\sigma}(t, \tau)\right\}, \sigma \in$ $\Sigma$, if for any $\tau \in \mathbb{R}, B \in \mathcal{B}(E)$, there exists $t_{0}=t_{0}(\tau, B)>\tau$ such that $\bigcup_{\sigma \in \Sigma} U_{\sigma}(t, \tau) B \subset B_{0}$ for all $t \geq t_{0}$.

A set $P$ belonging to $E$ is said to be uniformly (w.r.t. $\sigma \in \Sigma$ ) attracting set for the family of processes $U_{\sigma}(t, \tau), \sigma \in \Sigma$, if for an arbitrary fixed $\tau \in \mathbb{R}$ and any $B \in \mathcal{B}(E)$,

$$
\lim _{t \rightarrow \infty} \sup _{\sigma \in \Sigma} \operatorname{dist}_{E}\left(U_{\sigma}(t, \tau) B, P\right)=0
$$

where $\operatorname{dist}_{E}(X, Y)$ denotes the Hausdorff semi-distance for $X$ and $Y$ in $E$.

Definition 2.4. A closed set $\mathcal{A}_{\Sigma} \subset E$ is said to be the uniform attractor of a family of processes $\left\{U_{\sigma}(t, \tau)\right\}$ if it is uniformly (w.r.t. $\sigma \in \Sigma$ ) attracting (attracting property) and it is contained in any closed uniformly (w.r.t. $\sigma \in \Sigma)$ attracting set $\mathcal{A}^{\prime}$ of family of processes $\left\{U_{\sigma}(t, \tau)\right\}, \sigma \in \Sigma: \mathcal{A}_{\Sigma} \subseteq A^{\prime}$ (minimal property).

The uniformly (w.r.t. $\sigma \in \Sigma)$ w-limit set $\omega_{\tau, \Sigma}(B)$ of $\mathrm{B}$ is defined by $\omega_{\tau, \Sigma}(B)=\bigcap_{t \geq \tau} \overline{B_{t}}$, where $B_{t}=\bigcup_{\sigma \in \Sigma} \bigcup_{s>t} U_{\sigma}(s, \tau) B .\left\{U_{\sigma}(t, \tau)\right\}, \sigma \in \Sigma$, is said to be $\omega$-limit compact, if for any ${ }^{t \geq \tau} \in \mathcal{B}(E)$, it holds $\lim _{n \rightarrow \infty} K\left(B_{t}\right)=0$, where $\mathrm{K}$ is the Kuratowski measure of non-compactness . 
Theorem 2.2. ${ }^{[13]}$ Let $\Sigma$ be a subset of some Banach space, and let $T(t)$ be a continuous invariant $(T(t) \Sigma=\Sigma)$ semigroup on $\Sigma$ satisfying the translation identity. A family of processes $\left\{U_{\sigma}(t, \tau)\right\}, \sigma \in \Sigma$, possesses a compact uniform (w.r.t. $\sigma \in \Sigma$ ) attractor $\mathcal{A}_{\Sigma}$ satisfying

$$
\mathcal{A}_{\Sigma}=\omega_{0, \Sigma}\left(B_{0}\right)=\omega_{\tau, \Sigma}\left(B_{0}\right), \forall \tau \in \mathbb{R}
$$

if and only if it

(i) has a bounded uniformly (w.r.t. $\sigma \in \Sigma$ ) absorbing set $B_{0}$; and

(ii) is a uniformly (w.r.t. $\sigma \in \Sigma)$ w-limit compact.

Moreover, if $\Sigma$ is the weakly compact set in $\Xi$, the family of process $\left\{U_{\sigma}(t, \tau)\right\}, \sigma \in \Sigma$, with $\{T(h)\}_{h \geq 0}$ is $(E \times \Sigma, E)$ weakly continuous and satisfies (i)-(ii), then $\mathcal{A}_{\Sigma}$ satisfies

$$
\mathcal{A}_{\Sigma}=\mathcal{A}_{\Sigma_{0}}=\omega_{0, \Sigma}\left(B_{0}\right)=\bigcup_{\sigma \in \Sigma} \mathcal{K}_{\sigma}(0)
$$

Here $\Sigma_{0}$ is the weak closure of $\Sigma, \mathcal{K}_{\sigma}(0)$ is the section at $t=0$ of kernel $\mathcal{K}_{\sigma}$ of $\left\{U_{\sigma}(t, \tau)\right\}$ with symbol $\sigma \in \Sigma$.

We also need the following results.

Theorem 2.3. ${ }^{[14]}$ Assume that $p \geq q \geq 1$ and $\Omega \subset \mathbb{R}^{n}$ is bounded. Let $\left\{U_{\sigma}(t, \tau)\right\}, \sigma \in \Sigma$, be a family processes on $L^{p}(\Omega)$ and $L^{q}(\Omega)$, and satisfying the following conditions:

(i) $\left\{U_{\sigma}(t, \tau)\right\}$ is a uniform (w.r.t. $\left.\sigma \in \Sigma\right) \omega$-limit compact in $L^{q}(\Omega)$;

(ii) $\left\{U_{\sigma}(t, \tau)\right\}$ has a bounded absorbing set $B_{0}$ in $L^{p}(\Omega)$;

(iii) for any $\varepsilon>0$ and any bounded subset $B \subset L^{p}(\Omega)$, there exist positive constants $M=M(\varepsilon, B)$ and $T=T(\varepsilon, B)$ such that

$$
\int_{\Omega\left(\left|U_{\sigma}(t, \tau) u_{\tau}\right| \geq M\right)}\left|U_{\sigma}(t, \tau) u_{\tau}\right|^{p}<\varepsilon \text { for any } u_{\tau} \in B \text {, and } t \geq T, \sigma \in \Sigma .
$$

Then $\left\{U_{\sigma}(t, \tau)\right\}, \sigma \in \Sigma$ has a compact uniform (w.r.t. $\left.\sigma \in \Sigma\right)$ attractor in $L^{p}(\Omega)$.

\section{Existence of Uniform Attractor in $C_{L^{2}}$}

Lemma 3.1. Assume that the function a is locally lipschitz and satisfies (1.2), $f \in C(\mathbb{R})$ fulfills (1.3)-(1.4), $h \in L_{\text {loc }}^{2}\left(\mathbb{R} ; H^{-1}(\Omega)\right), g$ satisfies $(I)-(I I)$, and $\varphi \in C_{L^{2}}$. Then the weak solution of (1.1) continuously depends on the initial data.

Proof. Let $u=u\left(u \cdot ; \tau, \varphi_{1}\right)$ and $v=\left(v \cdot ; \tau, \varphi_{2}\right)$ be two solutions of (1.1) with respect to initial conditions $\left(u_{\tau}, h_{1}\right)$ and $\left(v_{\tau}, h_{2}\right)$, respectively. Denote by $w=u-v$ with $w(\tau)=u(\tau)-v(\tau)$, then from energy equality $(2.2)$ we have

$$
\begin{aligned}
\frac{1}{2} \frac{d}{d t}|w(t)|^{2}+a(l(u(t)))\|w(t)\|^{2} \\
=[a(l(v(t)))-a(l(u(t)))]((v(t), w(t)))+(f(u(t))-f(v(t)), w(t)) \\
\quad+\left(g\left(u_{t}\right)-g\left(v_{t}\right), w(t)\right)+<h_{1}(t)-h_{2}(t), w(t)>, \text { a.e. } t>\tau .
\end{aligned}
$$

Using (1.2),(1.4),(II) and the local lipschitz property of $a$, it yields

$$
\begin{aligned}
\frac{1}{2} \frac{d}{d t}|w(t)|^{2}+m\|w(t)\|^{2} & \leq L_{a}(R)|l\|w(t) \mid\| v(t)\|\| w(t) \| \\
& +\ell|w(t)|^{2}+L_{g}|w(t)|^{2}+\left\|h_{1}(t)-h_{1}(t)\right\|_{H^{-1}}\|w(t)\|,
\end{aligned}
$$

where $L_{a}(R)$ is the lipschitz constant of the function $a$ in $[-R, R]$. Thanks to the Young inequality, we get that

$$
\frac{d}{d t}|w(t)|^{2} \leq\left[\frac{1}{m} L_{a}^{2}(R)|l|^{2}\|v(t)\|^{2}+2 \ell\right]|w(t)|^{2}+2 L_{g}\left|w_{t}\right|^{2}+\frac{1}{m}\left\|h_{1}(t)-h_{2}(t)\right\|_{H^{-1}}^{2}
$$


Thus, we deduce that

$$
|w(t)|^{2} \leq e^{\int_{\tau}^{t}\left[\frac{1}{m} L_{a}^{2}(R) l^{2}\|v(s)\|^{2}+2 \ell\right] d s}\left[\left|w_{\tau}\right|^{2}+\int_{\tau}^{t}\left(\frac{1}{m}\left\|h_{1}(s)-h_{2}(s)\right\|_{H^{-1}}^{2}+2 L_{g}\left|w_{s}\right|^{2}\right) d s\right] .
$$

In particular, we get that

$$
\left|w_{t}\right|_{C_{L^{2}}} \leq e^{\int_{\tau}^{t}\left[\frac{1}{m} L_{a}^{2}(R) l^{2}\|v(s)\|^{2}+2 \ell\right] d s}\left[\left|w_{\tau}\right|^{2}+\frac{1}{m}\left\|h_{1}-h_{2}\right\|_{L_{l o c}^{2}\left(\mathbb{R} ; H^{-1}\right)}^{2}+2 L_{g} \int_{\tau}^{t}\left|w_{s}\right|_{C_{L^{2}}}^{2} d s\right] .
$$

By Gronwall's inequality from (3.5) we get that

$$
\begin{aligned}
\left|w_{t}\right|_{C_{L^{2}}} \leq & e^{\int_{\tau}^{t}\left[\frac{1}{m} L_{a}^{2}(R) l^{2}\|v(s)\|^{2}+2 \ell\right] d s}\left(\left|w_{s}\right|^{2}+\frac{1}{m}\left\|h_{1}(s)-h_{2}(s)\right\|_{L_{l o c}^{2}\left(\mathbb{R} ; H^{-1}\right)}^{2}\right) \\
& \times\left(1+e^{2 L_{g}(t-\tau) e} \int_{\tau}^{\int_{\tau}^{t}\left[\frac{1}{m} L_{a}^{2}(R) l^{2}\|v(s)\|^{2}+2 \ell\right] d s}\right),
\end{aligned}
$$

which implies that result.

We know from Theorem 2.1 that the solution of (1.1) can be represented by $U_{h}(t, \tau) \varphi$ with initial datum $\varphi$ belonging to $C\left([-k, 0] ; L^{2}(\Omega)\right)$ and time symbol $h$ belonging to $L_{l o c}^{2}\left(\mathbb{R} ; H^{-1}(\Omega)\right), U_{h}(t, \tau)$ : $C_{L^{2}} \longrightarrow C_{L^{2}}, t \geq \tau$ :

$$
U_{h}(t, \tau) \varphi=u_{t},
$$

where $u(\cdot)=u(\cdot ; \tau, \varphi)$ is the weak solution to (1.1), and by lemma 3.1, the process $\left\{U_{h}(t, \tau)\right\}$ is continuous in $C_{L^{2}}$.

Suppose that

$$
\|h\|_{L_{b}^{2}\left(\mathbb{R} ; H^{-1}(\Omega)\right)}=\sup _{t \in \mathbb{R}} \int_{t}^{t+1}\|h\|_{*}^{2} d s<+\infty .
$$

For fixed symbol $h_{0} \in L_{b}^{2}\left(\mathbb{R} ; H^{-1}(\Omega)\right)$, let $\Sigma={\overline{\left\{T(\nu) h_{0}(t), \nu \in \mathbb{R}\right\}}}^{L_{\text {loc }}^{2}\left(\mathbb{R} ; H^{-1}(\Omega)\right)}$. From proposition V.4.2 in $[12]$, we can obtain that

$$
\|h\|_{L_{b}^{2}\left(\mathbb{R} ; H^{-1}(\Omega)\right)} \leq\left\|h_{0}\right\|_{L_{b}^{2}\left(\mathbb{R} ; H^{-1}(\Omega)\right)}, \forall h \in \Sigma .
$$

Lemma 3.2. Under the assumptions of Lemma 3.1, the family of processes $\left\{U_{h}(t, \tau)\right\}, h \in \Sigma$, possesses a uniform (w.r.t. $h \in \Sigma$ ) absorbing set $B_{0}$ in $C_{L^{2}}$.

Proof. From (1.1), we get that

$$
\frac{1}{2} \frac{d}{d t}|u(t)|^{2}-(a(l(u(t))) \triangle u(t), u(t))=(f(u(t)), u(t))+\left(g\left(u_{t}\right), u(t)\right)+\langle h(t), u(t)\rangle .
$$

Using (1.2)-(1.4),(II) and the Young inequality, we obtain that

$$
\begin{aligned}
\frac{d}{d t}|u(t)|^{2} & +2(m-\mu)\|u(t)\|^{2}+2 \alpha_{2}\|u(t)\|_{L^{p}}^{p} \\
\leq & 2 c_{1}|\Omega|+2 L_{g}\left|u_{t}\right|_{C_{L^{2}}}|u(t)|+\frac{1}{2 \mu}\|h(t)\|_{*}^{2} \\
\leq & 2 c_{1}|\Omega|+2 L_{g}\left|u_{t}\right|_{C_{L^{2}}}^{2}+\frac{1}{2 \mu}\|h(t)\|_{*}^{2},
\end{aligned}
$$

where $0<\mu<m-\frac{L_{g}}{\lambda_{1}}$.

Dropping the third term on the left-hand side of (3.9), and using the Poincaré inequality and Gronwall's inequality, we can obtain that

$$
\begin{aligned}
|u(t)|^{2} \leq & |u(\tau)|^{2} e^{-2(m-\mu) \lambda_{1}(t-\tau)} \\
& \quad+\int_{\tau}^{t} e^{-2(m-\mu) \lambda_{1}(t-s)}\left(2 c_{1}|\Omega|+2 L_{g}\left|u_{s}\right|_{C_{L^{2}}}^{2}+\frac{1}{2 \mu}\|h(s)\|_{*}^{2}\right) d s .
\end{aligned}
$$


From the above inequality, we get that

$$
\begin{aligned}
e^{2(m-\mu) \lambda_{1} t}\left|u_{t}\right|_{C_{L^{2}}}^{2} \leq & e^{2(m-\mu) \lambda_{1} \tau}\left|u_{\tau}\right|_{C_{L^{2}}}^{2} \\
& +\int_{\tau}^{t} e^{2(m-\mu) \lambda_{1} s}\left(2 c_{1}|\Omega|+2 L_{g}\left|u_{s}\right|_{C_{L^{2}}}^{2}+\frac{1}{2 \mu}\|h(s)\|_{*}^{2}\right) d s .
\end{aligned}
$$

Using the Gronwall's inequality again, we get that, for any bounded set $B \subset C_{L^{2}}$, there exists $T_{1}=$ $T_{1}(B)>0$ such that

$$
\begin{aligned}
\left|u_{t}\right|_{C_{L^{2}}}^{2} \leq & e^{-\left[2(m-\mu) \lambda_{1}-2 L_{g}\right](t-\tau)}\left|u_{\tau}\right|_{C_{L^{2}}}^{2}+\int_{\tau}^{t} e^{-\left[2(m-\mu) \lambda_{1}-2 L_{g}\right](t-s)}\left(2 c_{1}|\Omega|+\frac{1}{2 \mu}\|h(s)\|_{*}^{2}\right) d s \\
\leq & e^{-2\left[(m-\mu) \lambda_{1}-L_{g}\right](t-\tau)}\left|u_{\tau}\right|_{C_{L^{2}}}^{2}+\frac{c_{1}|\Omega|}{(m-\mu) \lambda_{1}-L_{g}}+\frac{1}{2 \mu}\left[\int_{t-1}^{t}\|h(s)\|_{*}^{2} d s\right. \\
& \left.+e^{-\left[2(m-\mu) \lambda_{1}-2 L_{g}\right]} \int_{t-2}^{t-1}\|h(s)\|_{*}^{2} d s+e^{-\left[2(m-\mu) \lambda_{1}-L_{g}\right] \times 2} \int_{t-3}^{t-2}\|h(s)\|_{*}^{2} d s+\cdots\right] \\
\leq & e^{-2\left[(m-\mu) \lambda_{1}-L_{g}\right](t-\tau)}\left|u_{\tau}\right|_{C_{L^{2}}}^{2}+\frac{c_{1}|\Omega|}{(m-\mu) \lambda_{1}-L_{g}}+\frac{1}{2 \mu} \times \frac{1}{1-e^{-2\left[(m-\mu) \lambda_{1}-L_{g}\right]}} \\
& \times\left\|h_{0}\right\|_{L_{b}^{2}\left(\mathbb{R} ; H^{-1}(\Omega)\right)}^{2} \\
\leq & 2 \times\left[\frac{c_{1}|\Omega|}{(m-\mu) \lambda_{1}-L_{g}}+\frac{1}{2 \mu} \times \frac{1}{1-e^{-2(m-\mu) \lambda_{1}-L_{g}}}\left\|h_{0}\right\|_{L_{b}^{2}\left(\mathbb{R} ; H^{-1}(\Omega)\right)}^{2}\right] \\
\triangleq & \rho^{2}, \quad \forall t \geq \tau+T_{1} .
\end{aligned}
$$

Denote by $B_{0}=B_{C_{L^{2}}}(0, \rho)$, then $\left\{U_{h}(t, \tau)\right\}, h \in \Sigma$, has a bound uniformly $($ w.r.t. $h \in \Sigma)$ absorbing set $B_{0}$.

Denote $B_{1}=\bigcup_{h \in \Sigma} \bigcup_{\tau \in \mathbb{R}} U_{h}\left(\tau+T_{0}, \tau\right) B_{0}$, where $T_{0}>0$ is the uniform absorbing time of $B_{0}$, namely, $\bigcup_{h \in \Sigma} U_{h}\left(\tau+T_{0}, \tau\right) B_{0} \subset B_{0}$. Then $B_{1}$ is also the bounded uniform absorbing set.

Lemma 3.3. Under the assumptions of lemma 3.1, the family of processes $\left\{U_{h}(t, \tau)\right\}, h \in \Sigma$, is uniformly (w.r.t. $h \in \Sigma)$ w-limit compact.

Proof. Denote $U_{h_{n}}\left(t_{n}, \tau\right) \varphi^{n}=u^{n}\left(t_{n}, \tau, h_{n}, \varphi^{n}\right)=u^{n}(\cdot)$, where $\varphi^{n}=u_{\tau_{n}}^{n}$. In order to prove the family of processes $\left\{U_{h_{n}}\left(t_{n}, \tau\right)\right\}, h_{n} \in \Sigma$, is uniformly (w.r.t. $\left.h_{n} \in \Sigma\right)$ w-limit compact in $C_{L^{2}}$, we need to verify that for any sequence $\left\{u_{\tau_{n}}^{n}\right\} \subset B_{1},\left\{h_{n}\right\} \subset \Sigma$ and $\left\{t_{n}\right\} \subset \mathbb{R}_{\tau}$, the process $U_{h_{n}}\left(t_{n}, \tau\right) u_{\tau_{n}}^{n} \rightarrow u(t)$ in $C_{L^{2}}$ as $n \rightarrow \infty$.

Since $\left\{u_{\tau_{n}}^{n}\right\} \subset B_{1}$ and by lemma 3.2, we know that there $n_{1} \geq 1$, for any fixed $T>k, n \geq n_{1}$, it holds

$$
\left|u_{t}^{n}\right|_{C_{L^{2}}} \leq \rho^{2}, \forall t \in[\tau, \tau+T], n \geq n_{1}, \tau \in \mathbb{R}
$$

If we denote $y^{n}(t)=u^{n}(t+\tau)$ for all $t \in[0, T]$, we have that $\left\{y^{n}\right\}_{n \geq n_{1}}$ is uniformly bounded in $L^{\infty}\left(0, T ; L^{2}(\Omega)\right)$. Moreover, for each $n \geq n_{1}$, let $h$ be replaced by

$$
\widetilde{h}(t, \cdot)=h(t+\tau), \quad \forall t \in(0, T), \tau \in \mathbb{R} .
$$

Then by lemma 3.2 and (3.9), we have

$$
\left|y_{\tau}^{n}\right|_{L_{L^{2}}} \leq \rho^{2}
$$

and

$$
\begin{aligned}
& 2(m-\mu)\left\|y^{n}\right\|_{L^{2}\left(0, T ; H_{0}^{1}(\Omega)\right)}^{2}+2 \alpha_{2}\left\|y^{n}\right\|_{L^{p}\left(0, T ; L^{p}(\Omega)\right.}^{p} \\
& \leq 2 c_{1}|\Omega| T+2 L_{g} \rho^{2}+\frac{C}{2 \mu}\left\|h_{0}\right\|_{L_{b}^{2}\left(\mathbb{R} ; H^{-1}(\Omega)\right)}^{2}, \quad \forall n \geq n_{1} .
\end{aligned}
$$

As a consequence, $\left\{y^{n}\right\}_{n \geq n_{1}}$ is uniformly bounded in $L^{2}\left(0, T ; H_{0}^{1}(\Omega)\right) \cap L^{p}\left(0, T ; L^{p}(\Omega)\right)$. By $(1.3)$, we know $\left\{f\left(y^{n}\right)\right\}_{n \geq n_{1}}$ is uniformly bounded in $L^{q}\left(0, T ; L^{q}(\Omega)\right)$, where $\frac{1}{p}+\frac{1}{q}=1$, and by fact that $-\Delta$ is isometric isomorphism from $H_{0}^{1}(\Omega)$ to $H^{-1}(\Omega)$, we get that $\left\{\left(y^{n}\right)^{\prime}\right\}$ is uniformly bounded in $L^{2}\left(0, T ; H^{-1}(\Omega)\right)+$ 
$L^{q}\left(0, T ; L^{q}(\Omega)\right)$. Then by applying the Aubin-Lions lemma (see, e.g., Theorem 8.1 in [15]), there exists $y \in L^{2}\left(0, T ; H^{-1}(\Omega)\right) \cap L^{p}\left(0, T ; L^{p}(\Omega)\right) \cap C\left([0, T] ; L^{2}(\Omega)\right)$ and $y^{\prime} \in L^{2}\left(0, T ; H^{-1}(\Omega)\right)+L^{q}\left(0, T ; L^{q}(\Omega)\right)$ such that there exists a subsequence of $\left\{y^{n}\right\}_{n \geq n_{1}}$ (relabeled the same) and $\chi \in L^{q}\left(0, T ; L^{q}(\Omega)\right)$ such that

$$
\left\{\begin{array}{l}
y^{n} \stackrel{*}{\rightarrow} y \quad \text { weakly }- \text { star in } L^{\infty}\left(0, T ; L^{2}(\Omega)\right), \\
y^{n} \rightarrow y \quad \text { weakly in } L^{p}\left(0, T ; L^{p}(\Omega)\right), \\
y^{n} \rightarrow y \quad \text { weakly in } L^{2}\left(0, T ; H_{0}^{1}(\Omega)\right), \\
y^{n} \rightarrow y \quad \text { strongly in } L^{2}\left(0, T ; L^{2}(\Omega)\right), \\
\left(y^{n}\right)^{\prime} \rightarrow y^{\prime} \text { weakly in } L^{2}\left(0, T ; H^{-1}(\Omega)\right)+L^{q}\left(0, T ; L^{q}(\Omega)\right), \\
f\left(y^{n}\right) \rightarrow \mathcal{X} \text { weakly in } L^{q}\left(0, T ; L^{q}(\Omega)\right), \\
a\left(l\left(y^{n}\right)\right) \rightarrow a(l(y)) \text { weakly in } L^{2}\left(0, T ; H_{0}^{1}(\Omega)\right) .
\end{array}\right.
$$

Similar to the proof of Theorem 8.4 in [15], we can get that $f(y)=\mathcal{X}$. Since $\left\{y^{n}\right\}_{n \geq n_{1}}$ is bounded in $C\left([0, T] ; L^{2}(\Omega)\right)$, we deduce that for any sequence $s_{n} \subset[0, T]$ with $s_{n} \rightarrow s_{*}$, one has

$$
y^{n}\left(s_{n}\right) \rightarrow y\left(s_{*}\right) \quad \text { weakly in } L^{2}(\Omega) .
$$

On the other hand, from (I),(II), and (3.13) we have

$$
\int_{s}^{t}\left|g\left(y_{r}^{n}\right)\right|^{2} \leq C_{1}(t-s) \quad \forall 0 \leq s \leq t \leq T, n>n_{1}
$$

where $C_{1}>0$ is independent of $n$. This implies that there exists $\xi \in L^{2}\left(0, T ; L^{2}(\Omega)\right)$ such that

$$
g\left(y^{n}\right) \rightarrow \xi \quad \text { weakly in } L^{2}\left(0, T ; L^{2}(\Omega)\right),
$$

and therefore,

$$
\int_{s}^{t}|\xi(r)|^{2} d r \leq C_{1}(t-s) \quad \forall 0 \leq s \leq t \leq T .
$$

Hence, from above convergence we may deduce that $y$ is the unique weak solution of the equation

$$
\frac{\partial u}{\partial t}-a(l(u)) \triangle u=f(u)+\xi+\widetilde{h}(t)
$$

and satisfies $u(0)=y(0)$.

Using the energy equality, (3.16),(3.18) and the Young inequality, we can get the estimate

$$
\frac{1}{2}|z(s)|^{2} \leq \frac{1}{2}|z(r)|^{2}+\left(\frac{C_{1}}{2 m \lambda_{1}}+c_{1}|\Omega|\right)(t-s)+\frac{1}{2 m} \int_{s}^{t}\|h(r)\|_{*}^{2} d r \quad \forall \tau \leq r \leq s \leq t,
$$

where $z$ equals to $y^{n}$ or $y$.

Now we define the functions

$$
\begin{aligned}
J_{n}(t) & =\frac{1}{2}\left|y^{n}(t)\right|^{2}-\left(\frac{C_{1}}{2 m \lambda_{1}}+c_{1}|\Omega|\right) t-\frac{1}{2 m} \int_{\tau}^{t}\|h(r)\|_{*}^{2} d r, \\
J(t) & =\frac{1}{2}|y(t)|^{2}-\left(\frac{C_{1}}{2 m \lambda_{1}}+c_{1}|\Omega|\right) t-\frac{1}{2 m} \int_{\tau}^{t}\|h(r)\|_{*}^{2} d r .
\end{aligned}
$$

It follows that these maps $J_{n}$ and $J$ are continuous and non-increasing from $[\tau, T] \rightarrow \mathbb{R}$. Furthermore, we can obtain from (3.14) that

$$
J_{n}(t) \rightarrow J(t) \quad \text { a.e. } t \in(0, T) .
$$

If we prove that for any $\delta>0$

$$
y^{n} \rightarrow y \quad \text { strong in } C\left([\delta, T] ; L^{2}(\Omega)\right) \text {, }
$$


then we will get that $\left\{U_{h_{n}}\left(t_{n}, \tau\right)\right\}$ is relatively compact in $C_{L^{2}}$.

Suppose that there exists $0<\delta_{*}<T, \varepsilon_{*}>0$, and sequence $\left\{y^{m}\right\} \subset\left\{y^{n}\right\}_{n \geq n_{0}}, t_{m} \in\left[\delta_{*}, T\right]$ such that

$$
\left|y^{m}\left(t_{m}\right)-y\left(t_{*}\right)\right| \geq \varepsilon_{*}, \quad \forall m \geq 1 .
$$

First, we note that

$$
\left|y\left(t_{*}\right)\right| \leq \lim _{m \rightarrow \infty} \inf \left|y^{m}\left(t_{m}\right)\right|
$$

By (3.14), we get that for $t_{m} \subset\left[\delta_{*}, T\right]$, there holds

$$
\lim _{n \rightarrow \infty} J_{n}\left(t_{m}\right)=J\left(t_{m}\right)
$$

Fix $\varepsilon>0$. Since $J$ is continuous and non-increasing on $[0, T]$, we get that there exists $k(\varepsilon) \geq 1$ such that

$$
\left|J\left(t_{m}\right)-J\left(t_{*}\right)\right| \leq \frac{\varepsilon}{2}, \quad \forall m>k(\varepsilon)
$$

$\mathrm{By}(3.25)$ we get that there exists $n(\varepsilon) \geq 1$, for $m \geq n(\varepsilon)$, there holds

$$
\left|J_{n}\left(t_{m}\right)-J\left(t_{m}\right)\right| \leq \frac{\varepsilon}{2}
$$

Then, since all $J_{n}$ are non-increasing, we deduce that for all $m>m(\varepsilon)=\max \{k(\varepsilon), n(\varepsilon)\}$,

$$
\begin{aligned}
J_{n}\left(t_{n}\right)-J\left(t_{*}\right) & \leq J_{n}\left(t_{m}\right)-J\left(t_{*}\right) \\
& \leq\left|J_{n}\left(t_{m}\right)-J\left(t_{*}\right)\right| \\
& \leq\left|J_{n}\left(t_{m}\right)-J\left(t_{m}\right)\right|+\left|J\left(t_{m}\right)-J\left(t_{*}\right)\right| \\
& \leq \varepsilon, \quad \forall m \geq m(\varepsilon) .
\end{aligned}
$$

Since $\varepsilon>0$ is arbitrary, we conclude that

$$
\lim _{m \rightarrow+\infty} \sup \left|J_{m}\left(t_{m}\right)\right| \leq J\left(t_{*}\right)
$$

which implies that

$$
\lim _{m \rightarrow \infty} \sup \left|y^{m}\left(t_{m}\right)\right| \leq y\left(t_{*}\right) .
$$

From this inequality and (3.14)and (3.15), we have that $y^{n}\left(t_{n}\right) \rightarrow y\left(t_{*}\right)$ strongly in $C_{L^{2}}$, which is contradict with $(3,23)$. This completes the proof.

By Lemma 3.1-3.3 and applying Theorem 2.2, we obtain the following result:

Theorem 3.4. Assume that $f$ satisfies (1.3)-(1.4) and $g$ satisfies (I)-(II), and $h \in \Sigma$. Then there exists a compact uniform (w.r.t, $h \in \Sigma$ ) attractor for the family of processes $U_{h}(t, \tau), h \in \Sigma$, in $C_{L^{2}}$. Moreover, it holds

$$
A=\omega_{0, \Sigma}\left(B_{0}\right)=\omega_{\tau, \Sigma}\left(B_{0}\right)=\bigcup_{h \in \Sigma} \mathcal{K}_{h}(s), \quad \forall s \in \mathbb{R}
$$

where $B_{0}$ is the bound uniformly (w.r.t. $h \in \Sigma$ ) absorbing set in $C_{L^{2}}$ and $\mathcal{K}_{\sigma}(s)$ is the section at $t=s$ of kernel $\mathcal{K}_{\sigma}$.

\section{Existence of Uniform Attractor in $C_{L^{p}}$}

Let $h(t)$ be translation bounded in $L_{\text {loc }}^{2}\left(\mathbb{R} ; L^{2}(\Omega)\right)$, i.e.,

$$
\|h\|_{L_{b}^{2}\left(\mathbb{R} ; L^{2}(\Omega)\right)}=\sup _{t \in \mathbb{R}} \int_{t}^{t+1}|h(s)|^{2} d s<+\infty .
$$


Denote by

$$
\Sigma_{1}=\overline{\left\{T(\nu) h_{0}(t), \nu \in \mathbb{R}\right\}} L_{\text {loc }}^{2, \omega}\left(\mathbb{R} ; L^{2}(\Omega)\right),
$$

the closure of the set $\left\{T(\nu) h_{0}(t), \nu \in \mathbb{R}\right\}$ in the topology of $L_{\text {loc }}^{2, \omega}\left(\mathbb{R} ; L^{2}(\Omega)\right)$. As the proof of lemma 3.2 , we have

Lemma 4.1. Under the assumption of lemma 3.1, the family of process $\left\{U_{h}(t, \tau)\right\}, h \in \Sigma_{1}$ has a uniformly absorbing set in $C_{L^{p}}, p \geq 2$, that is, for any bounded set $B \subset C_{L^{2}}$, there exists a positive constant $\rho_{p}$ and $t_{0}=t_{0}(\tau, B) \geq \tau$ such that

$$
\left\|U_{h}(t, \tau) u_{\tau}\right\|_{C_{L^{p}}} \leq \rho_{p}, \text { for any } t \geq t_{0}, u_{\tau} \in B, h \in \Sigma_{1} .
$$

Proof. Multiplying (1.1) by $|u|^{p-2} u$ and integrating by parts, using (1.2)-(1.3) and (I)-(II), we get that

$$
\begin{aligned}
\frac{1}{p} \frac{d}{d t}\|u\|_{L^{p}}^{p} & +\left.\left.\frac{4 m(p-1)}{p^{2}} \int_{\Omega}|\nabla| u\right|^{\frac{p}{2}}\right|^{2} d x+\alpha_{2} \int_{\Omega}|u|^{2 p-2} d x \\
& \leq c_{1}|\Omega|+\int_{\Omega} g\left(u_{t}\right)|u|^{p-2} u d x+\int_{\Omega} h(t)|u|^{p-2} u d x \\
& \leq c_{1}|\Omega|+L_{g}\left|u_{t}\right|_{C_{L^{2}}} \int_{\Omega}|u|^{p-1} d x+\int_{\Omega}|h(t)||u|^{p-1} d x \\
& \leq c_{1}|\Omega|+\frac{|\Omega|}{2 \alpha_{1}} L_{g}^{2}\left|u_{t}\right|_{C_{L^{2}}}^{2}+\frac{1}{2 \alpha_{1}}|h(t)|^{2}+\alpha_{2} \int_{\Omega}|u|^{2 p-2} d x
\end{aligned}
$$

which yields

$$
\frac{d}{d t}\|u\|_{L^{p}}^{p} \leq p\left(c_{1}|\Omega|+\frac{|\Omega|}{2 \alpha_{1}} L_{g}^{2}\left|u_{t}\right|_{C_{L^{2}}}^{2}\right)+\frac{p}{2 \alpha_{1}}|h(t)|^{2}
$$

By (3.9), we get that for any $u_{\tau} \in B_{1}$, there holds

$$
\begin{aligned}
\int_{t-1}^{t}\|u(t)\|_{L^{p}}^{p} d t & \leq \frac{1}{2 \alpha_{2}}\left[2 c_{1}|\Omega|+\left(2 L_{g}+1\right) \rho^{2}\right]+\frac{C}{4 \alpha_{2} \mu} \int_{\tau}^{\tau+1}|h(s)|^{2} d s \\
& \leq \frac{1}{2 \alpha_{2}}\left[2 c_{2}|\Omega|+\left(2 L_{g}+1\right) \rho^{2}\right]+\frac{C}{4 \alpha_{2} \mu}\left|h_{0}\right|_{L_{b}^{2}\left(\mathbb{R} ; L^{2}(\Omega)\right)}^{2}, \forall t \in \mathbb{R} .
\end{aligned}
$$

Therefore, using uniform Gronwall Lemma (see, e.g., Lemma III 1.1 in [16]), it follows from (4.3)-(4.4) that

$$
\|u(t)\|_{L^{p}}^{p} \leq\left(\frac{c_{2}}{\alpha_{2}}+p c_{1}\right)|\Omega|+\left(\frac{2 L_{g}+1}{2 \alpha_{2}}+\frac{p|\Omega|}{2 \alpha_{1}}\right) \rho^{2}+\left(\frac{p}{2 \alpha_{1}}+\frac{C}{4 \alpha_{2} \mu}\right)\left|h_{0}\right|_{L_{b}^{2}\left(\mathbb{R} ; L^{2}(\Omega)\right)}, \forall t \in \mathbb{R}
$$

which implies that

$$
\begin{aligned}
\|u\|_{C_{L^{p}}}^{p} & \leq\left(\frac{c_{2}}{\alpha_{2}}+p c_{1}\right)|\Omega|+\left(\frac{2 L_{g}+1}{2 \alpha_{2}}+\frac{p|\Omega|}{2 \alpha_{1}}\right) \rho^{2}+\left(\frac{p}{2 \alpha_{1}}+\frac{C}{4 \alpha_{2} \mu}\right)\left|h_{0}\right|_{L_{b}^{2}\left(\mathbb{R} ; L^{2}(\Omega)\right)} \\
& \triangleq \rho_{p}^{p}, \quad \forall t \geq \tau, h \in \Sigma_{1} .
\end{aligned}
$$

The proof is completed.

Definition 4.1. A function $\phi \in L_{l o c}^{2}(\mathbb{R} ; E)$ is said to be normal, if for any $\varepsilon>0$ there exists $\eta>$ such that $\sup _{t \in \mathbb{R}} \int_{t}^{t+\eta}\|\varphi\|_{E}^{2} d s \leq \varepsilon$.

Denote by $L_{n}^{2}(\mathbb{R} ; E)$ the set of all normal functions in $L_{\text {loc }}^{2}(\mathbb{R} ; E)$. It is easy to see that $L_{n}^{2}(\mathbb{R} ; E) \subset$ $L_{b}^{2}(\mathbb{R} ; E)$.

Lemma 4.2. ${ }^{[11]}$ If $\varphi_{0} \in L_{n}^{2}(\mathbb{R} ; E)$, then for any $\tau \in \mathbb{R}$,

$$
\lim _{\gamma \rightarrow+\infty} \sup _{t \geq \tau} \int_{\tau}^{t} e^{-\gamma(t-s)}\|\varphi(s)\|_{E}^{2} d s=0
$$


uniformly w.r.t. $\varphi \in H\left(\varphi_{0}\right)$, where $H\left(\varphi_{0}\right)=\left\{\varphi_{0}(t+h) \mid h \in \mathbb{R}\right\}$.

Theorem 4.3. If $h$ is normal in $L_{\text {loc }}^{2}\left(\mathbb{R} ; L^{2}(\Omega)\right)$, a is locally lipschitz and satisfies (1.2), $f \in C(\mathbb{R})$ satisfies (1.3)-(1.4) and $g$ satisfies (I)-(II), then the family of processes corresponding to problem (1.1) possess a compact uniform (w.r.t. $h \in \Sigma_{1}$ ) attractor $\mathcal{A}=\omega_{\tau, \Sigma_{1}}\left(B_{2}\right)$ in $C_{L^{p}}, p \geq 2$, which uniformly (w.r.t. $h \in \Sigma_{1}$ ) attracts every bounded set in $C_{L^{2}}$ in the norm of $C_{L^{p}}$, where $B_{2}$ is a bounded uniformly (w.r.t. $h \in \Sigma_{1}$ ) absorbing set in $C_{L^{p}}$.

Proof. By lemma 4.1, we know that $\left\{U_{h}(t, \tau)\right\}$ processes a uniform absorbing set $B_{2}$ in $C_{L^{p}}$. Now let us verify that $\left\{U_{h}(t, \tau)\right\}$ is a uniform $w$-limit compact in $C_{L^{p}}$. Let

$$
(u-M)_{+}=\left\{\begin{array}{l}
u-M, \quad u \geq M \\
0, \quad u \leq M
\end{array}\right.
$$

For any fixed $h \in \Sigma_{1}$, multiplying (1.1) by $\left|(u-M)_{+}\right|^{p-1}$ and using (1.2), we have

$$
\begin{aligned}
& \frac{1}{p} \frac{d}{d t}\left\|(u-M)_{+}\right\|_{p}^{p}+m(p-1) \int_{\Omega}\left|\nabla(u-M)_{+}\right|^{2}\left|(u-M)_{+}\right|^{p-2} d x \\
& \quad \leq\left.\int_{\Omega} f(u)(u-M)_{+}\right|^{p-1} d x+\int_{\Omega} g\left(u_{t}\right)(u-M)_{+}^{p-1} d x+\int_{\Omega} h(t)(u-M)_{+}^{p-1} d x .
\end{aligned}
$$

Set $\Omega_{1}=\Omega(u(t) \geq M)$, we have

$$
\begin{aligned}
& \frac{1}{p} \frac{d}{d t}\left\|(u-M)_{+}\right\|_{p}^{p}+m(p-1) \int_{\Omega_{1}}\left|\nabla(u-M)_{+}\right|^{2}\left|(u-M)_{+}\right|^{p-2} d x \\
& \leq \int_{\Omega_{1}} f(u)(u-M)_{+}^{p-1} d x+\int_{\Omega_{1}} g\left(u_{t}\right)(u-M)_{+}^{p-1} d x+\int_{\Omega_{1}} h_{1}(t)(u-M)_{+}^{p-1} d x .
\end{aligned}
$$

By(1.3), we can take $M$ large enough such that

$$
f(u) \leq-\alpha_{2}|u|^{p-1}, \text { in } \Omega_{1} .
$$

Therefore, we get that

$$
\begin{aligned}
& \frac{1}{p} \frac{d}{d t}\left\|(u-M)_{+}\right\|_{p}^{p}+m(p-1) \int_{\Omega_{1}}\left|\nabla(u-M)_{+}\right|^{2}(u-M)_{+}^{p-2} d x \\
& \quad+\alpha_{2} \int_{\Omega}|u|^{p-1}(u-M)_{+}^{p-1} d x \leq \int_{\Omega_{1}} g\left(u_{t}\right)(u-M)_{+}^{p-1} d x+\int_{\Omega_{1}} h(t)(u-M)_{+}^{p-1} d x .
\end{aligned}
$$

Since

$$
\begin{aligned}
\int_{\Omega_{1}} g\left(u_{t}\right)(u-M)_{+}^{p-1} d x & \leq \int_{\Omega_{1}} L_{g}\left|u_{t}\right|_{C_{L^{2}}}(u-M)_{+}^{p-1} \\
& \leq \frac{L_{g}^{2} \rho^{2}\left|\Omega_{1}\right|}{\alpha_{2}}+\frac{\alpha_{2}}{4} \int_{\Omega_{1}}(u-M)_{+}^{2 p-2} d x
\end{aligned}
$$

and

$$
\int_{\Omega_{1}} h(t)(u-M)_{+}^{p-1} d x \leq \frac{1}{\alpha_{2}} \int_{\Omega_{1}}|h(t)|^{2} d x+\frac{\alpha_{2}}{4} \int_{\Omega_{1}}(u-M)_{+}^{2 p-2} d x .
$$

It follows from (4.10)-(4.12) that

$$
\frac{1}{p} \frac{d}{d t}\left\|(u-M)_{+}\right\|_{p}^{p}+\frac{\alpha_{2}}{2} \int_{\Omega_{1}}|u|^{p-1}(u-M)_{+}^{p-1} d x \leq \frac{L_{g}^{2} \rho^{2}\left|\Omega_{1}\right|^{2}}{\alpha_{2}}+\frac{1}{\alpha_{2}} \int_{\Omega_{1}}|h(t)|^{2} d x .
$$

Since $u \geq M$ in $\Omega_{1}$, there holds

$$
|u|^{p-2}(u-M)_{+} \leq|u|^{p-1} .
$$


Thus,

$$
\frac{d}{d t}\left\|(u-M)_{+}\right\|_{p}^{p}+\frac{p \alpha_{2} M^{p-2}}{2} \int_{\Omega_{1}}\left|(u-M)_{+}^{p}\right| d x \leq p \frac{L_{g}^{2} \rho^{2}\left|\Omega_{1}\right|^{2}}{\alpha_{2}}+\frac{p}{\alpha_{2}} \int_{\Omega_{1}}|h(t)|^{2} d x .
$$

Therefore, by Gronwall's inequality, we get that

$$
\left\|(u-M)_{+}(t)\right\|_{p}^{p} \leq\left\|(u-M)_{+}(T)\right\|_{p}^{p} e^{-\lambda(t-T)}+\frac{p L_{g}^{2} \rho^{2}\left|\Omega_{1}\right|}{\lambda \alpha_{2}}+\frac{p}{\alpha_{2}} \int_{T}^{t} e^{-\lambda(t-s)}|h(t)|^{2} d s,
$$

where $\lambda=\frac{p \alpha_{2} M^{p-2}}{2}, T=T(B)$.

From lemma 4.2 , for any $\varepsilon>0$, we can take $M_{1}$ large enough such that

$$
\begin{gathered}
\frac{p}{\alpha_{2}} \int_{T}^{t} e^{-\lambda(t-s)}|h(t)|^{2} d s \leq \frac{\varepsilon}{3}, \text { for } M \geq M_{1}, \\
\left\|(u-M)_{+}(T)\right\|_{p}^{p} e^{-\lambda(t-T)} \leq \rho_{p}^{p} e^{-\lambda(t-T)} \leq \frac{\varepsilon}{3}, \quad \forall M \geq M_{1},
\end{gathered}
$$

and

$$
\frac{p L_{g}^{2} \rho^{2}\left|\Omega_{1}\right|}{\lambda \alpha_{2}} \leq \frac{\varepsilon}{3}
$$

Combing with (4.16)-(4.18), we deduce that

$$
\int_{\Omega(u(t) \geq M)}\left\|(u-M)_{+}\right\|_{p}^{p} d x \leq \varepsilon, \text { for any } t>t_{B}, h \in \Sigma_{1}, M \geq M_{1} .
$$

Similarly, multiplying (1.1) by $\left|(u+M)_{-}\right|^{p-2}(u+M)_{-}$, we deduce that there exists $M_{2}>0$ such that

$$
\int_{\Omega_{1}(u(t) \leq-M)}\left\|(u+M)_{-}\right\|_{p}^{p} d x \leq \varepsilon, \text { for any } t>t_{B}, h \in \Sigma_{1}, M \geq M_{2} .
$$

where

$$
(u+M)_{-}=\left\{\begin{array}{l}
u+M, \quad u \leq-M \\
0, \quad u \geq-M
\end{array}\right.
$$

Set $M_{3}=\max \left(M_{1}, M_{2}\right)$, we get that

$$
\int_{\Omega_{1}\left(|u(t)| \geq M_{3}\right)}\left\|\left(|u(t)|-M_{3}\right)\right\|_{p}^{p} d x \leq 2 \varepsilon, \text { for } t>t_{B}, h \in \Sigma_{1},
$$

and then

$$
\begin{aligned}
\int_{\Omega_{1}\left(|u(t)| \geq 2 M_{3}\right)} & \|u(t)\|_{p}^{p} d x \\
& =\int_{\Omega_{1}\left(|u(t)| \geq 2 M_{3}\right)}\left(\left(|u(t)|-M_{3}\right)+M_{3}\right)^{p} d x \\
& \leq 2^{p-1} \int_{\Omega_{1}\left(|u(t)| \geq 2 M_{3}\right)}\left(|u(t)|-M_{3}\right)^{p} d x+\int_{\Omega_{1}\left(|u(t)| \geq 2 M_{3}\right)} M_{3}^{p} d x \\
& \leq 2^{p-1} \int_{\Omega_{1}\left(|u(t)| \geq 2 M_{3}\right)}\left(|u(t)|-M_{3}\right)^{p} d x+\int_{\Omega_{1}\left(u(t) \geq M_{3}\right)}\left(|u(t)|-M_{3}\right)^{p} d x \\
& \leq 2^{p+1} \varepsilon .
\end{aligned}
$$

Therefore

$$
\left\|u_{t}\right\|_{C_{L}\left(|u(t)| \geq 2 M_{3}\right)} \leq 2^{1+\frac{1}{p}} \varepsilon^{\frac{1}{p}} .
$$

By Theorem 2.3 and 3.4, we get the result. 
Acknowledgments. The authors thank a lot to the reviewers, and with their help, the version of the paper can be improved greatly. This work was supported by the NSFC grant (No. 11571092).

\section{References}

1. S.B. de Menezes, Remarks on weak solutions for a nonlocal parabolic problem, Int.J.Math.Math. Sciences 2006 (2006)1-10.

2. S.M. Zheng, M. Chipot, Asymptotic behavior of solutions to nonlinear parabolic equations with nonlocal terms, Asymptotic Anal.45(2005)301-312.

3. M. Chipot, B. Lovat, On the asymptotic behaviour of some nonlocal problems, Positivity 3 (1999), 65-81.

4. J. Garcia-Luengo, P.Marin-Rubio, J. Real, Pullback attractors for 2D Navier-Stokes equations with delays and their regularity, Adv.Nonlinear Stud.13(2013)331-357.

5. A. Andami Ovono, Asymptotic behavior for a diffusion equation governed by nonlocal interaction, Electronic J.Differential.Equations 17(2010)1555-1666.

6. M. Anguiano, P.E. Kloeden, T. Lorenz, Asymptotic behaviour of nonlocal reaction diffusion equations, Nonlinear Anal.73(2010)3044-3057.

7. T. Caraballo, M. Herrera-Cobos, P. Marin-Rubio, Long time behavior of a nonautonomous parabolic equation with nonlocal diffusion and sublinear terms, Nonlinear Anal.121(2015)3-18.

8. W.W. Chang, X.J. Li, Dynamical behavior of non-autonomous parablic equation with nonlocal diffusion, J.Sci.Tech.Univ.Henan(Nat.Sci.)(Chinese) 37(5)(2016)77-82.

9. M. Chipot, L. Molinet, Asymptotic behaviour of some nonlocal diffusion problems, Applicable Anal. 80(2001)279-315.

10. J. Garcia-Luengo, P.Marin-Rubio, Reaction-diffusion equations with non-autonomous force in $H^{-1}$ and delays under measurablity conditions on the driving delay term, J.Math.Anal.Appl., 417(2014) 80-95.

11. H.M. Wei, X.Z. Li, M. Martcheva, An epidemic model of a vector-borne disease with direct transmis- sion and time delay, J.Math.Anal.Appl.2(342)(2008)895-908.

12. V.V. Chepyzhov, M.I.Vishik, Attractors for Equations of Mathematical Physics, Amer. Math. Soc. Colloq. Province, RI,2002.

13. S.S. Lu, H.Q. Wu, C.K. Zhong, Attractors for nonautonomous 2D navier-stokes equations with nor- mal external forces,Discrete.Cont.Dyn.Syst.13(3)(2005)701-709.

14. H.T. Song, C.K.Zhong, Attractors of non-autonmous reaction-diffusion equation in $L^{p}$, Nonlinear Anal.(68)2008,1890-1897.

15. J.C. Robinson, Infinite-dimensional Systems an Introduction to Dissipative Parabolic PDEs and the Theory of Global Attractors,Cambridge University Press, 2001.

16. R. Temam, Infinite-dimension Dynamical Systems in Mechanics and Physics, Springer, New York, 1997. 